ORIGINAL ARTICLE

\title{
A congested football calendar and the wellbeing of players: correlation between match exposure of European footballers before the World Cup 2002 and their injuries and performances during that World Cup
}

\author{
J Ekstrand, M Waldén, M Hägglund
}

Br J Sports Med 2004;38:493-497. doi: 10.1136/bjsm.2003.009134

\begin{abstract}
Objectives: To investigate the correlation between exposure of footballers in European clubs to match play in the months before the World Cup 2002 and their injuries and performances during that World Cup. Methods: The team doctors at 11 of the best football clubs in Europe prospectively recorded players' exposure and injuries during the 2001-2002 season (July 2001-May 2002). Sixty five players participated in the World Cup in Korea/Japan (June 2002). During the World Cup, the clubs reported injuries sustained by these players, and their performance was evaluated by three international experts. Results: The number of team matches during the season varied between 40 and 76 for the different countries involved. The individual player had a mean of 36 matches during the season. Top players played more matches, especially during the final period of the season. Players who participated in the World Cup played more matches during the season than those who did not (46 v33 matches). World Cup players did not show any increased risk of injury during the season. About $29 \%$ incurred injuries during the World Cup, and $32 \%$ performed below their normal standard. The players who underperformed had played more matches during the 10 weeks before the World Cup than those who performed better than expected (12.5 $v 9, \mathrm{p}<0.05)$. Twenty three (60\%) of the 38 players who had played more than one match a week before the World Cup incurred injuries or underperformed during the World Cup.

Conclusions: There is considerable variation in the number of matches played per season in European professional football leagues. Top level players are obliged to play many matches especially during the final period of the season.
\end{abstract}

See end of article for authors' affiliations

Correspondence to: Professor Ekstrand, Department of Social Medicine and Public Health Science, Linköping University, Linköping, Sweden; jan.ekstrand@ telia.com

Accepted

25 November 2003
$\mathrm{T}$ he overall level of injury for a professional footballer has been reported to be about 1000 times higher than that of a high risk industrial worker. ${ }^{1-5}$ A research group at the Football Association of England evaluated the risk and pattern of injuries in professional English football. ${ }^{1-6}$ The results showed that each injury caused, on average, absence from four matches, and that each week about $10 \%$ of a squad was unable to train because of injury. ${ }^{4}$ The financial loss attributable to absence of injured players during the 1999/ 2000 season in the English football leagues (92 clubs) was calculated to be about 125 million euros, an average of 1.4 million euros per team. ${ }^{4}$

Many football injuries can be prevented if appropriate prophylactic measures are taken..$^{7-10}$ Ekstrand et a l $^{9}$ published the first prospective randomised intervention study on amateur teams in 1982 showing that the rate of injury was $75 \%$ lower for the intervention teams than for the control teams. These findings were recently confirmed in an intervention study on amateur youth players performed by the F-Marc group, a research group within FIFA (Fédération Internationale de Football Associations). ${ }^{10}$

The mechanisms behind football injuries are complex and multifactorial. ${ }^{11-16}$ The way in which the season is planned is important with regard to injuries. ${ }^{13}$ Ekstrand et al ${ }_{1}^{17}$ studying the relation between training and matches at amateur levels, found that a high training/match quotient with many training sessions in relation to the number of matches played gave greater success and fewer injuries. However, this relation has not been studied at the professional level.

UEFA (Union des Associations Europeénnes de Football) has expressed its concern over the demands being placed on modern footballers and the translation of these physical and mental demands into injury syndromes. UEFA therefore initiated a research project specifically aimed at evaluating the exposure to football and the risk of injury of top level footballers in Europe. As this study provided information on exposure and injuries for many of the top European clubs during the season preceding the World Cup, players who participated were also followed during the World Cup period.

The aim of this study was to evaluate the correlation between exposure of footballers in top European clubs to match play during the season before the World Cup 2002 and the injuries and performance of these players during that competition.

\section{MATERIAL AND METHODS}

Fourteen of the top European clubs (clubs that have participated at the highest level in Europe over the last decade) were selected by UEFA and invited to take part in the study. We contacted the clubs and sent them information about the study. Before the study began, each team doctor was invited to a meeting for further information about study details. To avoid variation in data collection, great effort was put into standardising study details, such as the definition of injury, who determines when a player is fully rehabilitated after an injury, what should be regarded as a training session, etc. $^{18}$ Each club selected a contact person who was responsible for collecting data from the club and forwarding the information to the study group.

One team declined participation and two teams were excluded from the study because of insufficient data. The following 11 teams agreed to participate, delivered complete 
data, and were thus included: Arsenal FC (England); Manchester United FC (England); Paris Saint-Germain FC (France); Stade Rennais FC (France); RC Lens (France); AC Milan (Italy); Juventus FC (Italy); Internazionale FC (Italy); AFC Ajax (Netherlands); PSV Eindhoven (Netherlands); Real Madrid CF (Spain).

\section{Inclusion/exclusion criteria}

All contracted players in the A teams during the first month of the study (July 2001) were invited to participate in the study. Players contracted to the teams after July were not included. Players with an injury at the start of the study were included but this initial injury was not included in the injury statistics.

The players were informed about the study and accepted participation by signing an informed consent form.

\section{Data collection}

The clubs were provided with attendance record forms. The club contact person was responsible for completing this form with data about the players' attendance at training sessions and matches. Exposure times were registered in minutes for each individual player in order to base the incidence of injury on real exposure time. The attendance records included all training sessions and matches for the A team. Only coach directed sessions that included physical activity were recorded.

If selected players participated in training sessions and matches outside those for the A team-for example, B team, youth matches, or training sessions or matches for different national teams-this information was also included. To assess the risk of injury and exposure of the individual players in each team, it was important to include exposure to national team participation and the injuries that occurred as a result thereof. The contact person at each club therefore included exposure to matches and training sessions with the national team in the attendance records and, in the event of injury, filled in an injury card.

\section{Definition of injury}

All injuries were recorded on a special card. A recordable injury was defined as one that occurred during a scheduled match or training session and caused the player to miss the next match or training session.

\section{Rehabilitation}

A player was considered injured if he could not fully participate in all parts of a collective training session and was considered fully rehabilitated when he had full clearance from the club's medical officer.

\section{Study period}

The study covered the period from July 2001 to July 2002 and included the normal European football season (July 2001 to May 2002) as well as the World Cup 2002 in Korea/Japan (June 2002).

\section{Participation in the World Cup 2002}

A total of 266 players were included in the study. Sixty five of these played matches in the World Cup in Korea/Japan. Six other players were selected for the World Cup but, as they did not play in any match, evaluation of their performance was impossible and they were placed in the non-World Cup player group. Table 1 presents anthropometric data of the players for the two groups.

The clubs reported injuries sustained by these players during the event and during the preparation period with their national team before the World Cup (15-30 May). The
Table 1 Anthropometric data for the 65 footballers who participated and the 201 players who did not participate in World Cup matches in Korea and Japan 2002

\begin{tabular}{lcc}
\hline & $\begin{array}{l}\text { World Cup } \\
\text { players }\end{array}$ & $\begin{array}{l}\text { Non-World Cup } \\
\text { players }\end{array}$ \\
\hline No of players & 65 & 201 \\
Age (years) & 26 & 26 \\
Height $(\mathrm{cm})$ & 181 & 181 \\
Weight $(\mathrm{kg})$ & 78 & 78 \\
\hline
\end{tabular}

exposure to training, however, was not recorded during this period.

The performance of the players during the World Cup was evaluated by a group of three international experts (international coaches and former coaches of national teams in Europe). These experts were given a list of the 65 players and asked to assess their performance according to one of three levels:

- Overperformance (the player performed above his normal standard)

- Normal performance (the player performed as expected)

- Underperformance (the player performed below his normal standard)

\section{Analyses}

Comparisons were made between the World Cup players and the players who did not participate in the World Cup (nonWorld Cup players). The groups were compared for qualitative variables using the $\chi^{2}$ test, or Fisher's exact test for small numbers. Owing to a non-normal distribution, quantitative variables were compared between groups using the MannWhitney U test for unpaired comparisons and the Wilcoxon signed rank test for paired group comparisons. The significance level was set at $\mathrm{p}<0.05$. Results are expressed as mean (SD) and 95\% confidence interval (95\% CI).

\section{RESULTS}

Exposure and risk of injury during the whole season Exposure for the teams

The overall exposure to football during the full season for the 11 teams was 70000 hours (58000 training hours and 12000 match hours).

Each team had an average of 280 activities (training sessions and matches) during the season. The number of matches, however, differed between the various countries, ranging from 40 to 76 matches. The teams that played more matches obviously had less time to devote to training sessions.

\section{Exposure for individual players}

Each of the 266 players participated in a mean of 175 training sessions and 36 matches during the season. Table 2 shows exposures for the World Cup players and the non-World Cup players. The 65 players who later participated in the World Cup (June 2002) played significantly more matches during the ordinary season (July 2001-May 2002) than the 201 players who did not (46 (13) v 33 (16), p<0.001). There was no difference between the two groups with regard to the number of training sessions during the season.

\section{Risk of injury}

Table 3 shows the injury incidences for the World Cup and non-World Cup players during the season. The World Cup 


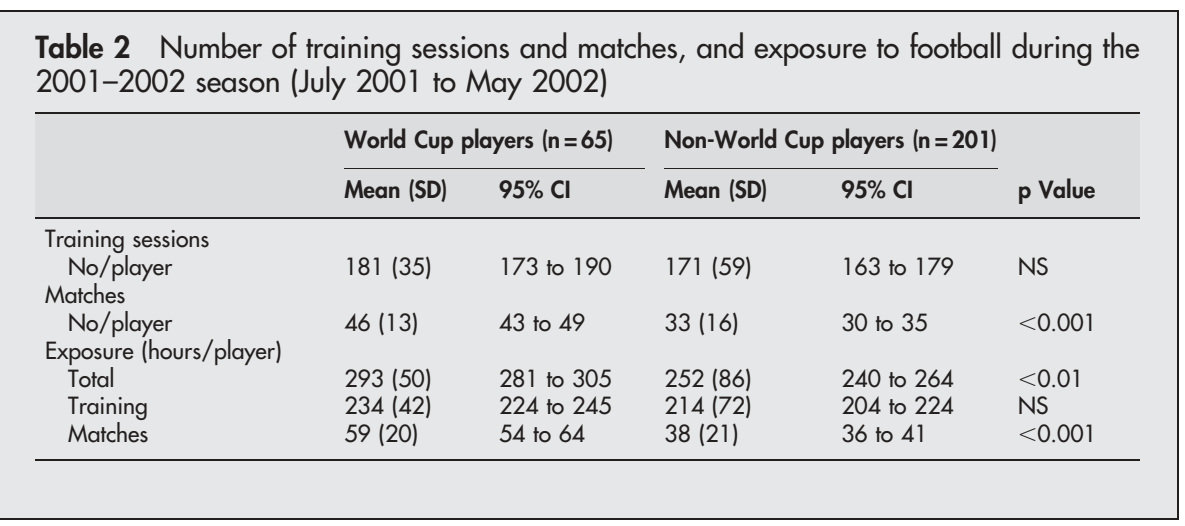

players had a lower risk of training injury $(3.2 v 5.5$ per 1000 hours of exposure, $\mathrm{p}<0.01$ ), whereas, during match play, there was no difference between the groups.

\section{Exposure and risk of injury during the final period of the season compared with the rest of the season}

The 266 players played a mean of 0.8 matches a week during the season (July 2001- May 2002). There was no difference in the number of matches played a week during the last three months of the season compared with the first eight months.

Table 4 presents the exposure to football per week during the last 10 weeks of the 2001-2002 season (March 2002 to 15 May 2002) and the first 36 weeks of the season (July 2001 to 28 February 2002). The 65 World Cup players played significantly more matches per week during the last 10 weeks of the season than during the first 36 weeks ( $1.12 v 0.97$, $\mathrm{p}<0.01)$. The 201 non-World Cup players on the other hand played significantly fewer matches per week during the last 10 weeks compared with the rest of the season $(0.66 v 0.72$, $\mathrm{p}<0.05$ ).

The World Cup players participated in four training sessions a week throughout the season; no difference was found between the first and last period. The non-World Cup players participated in more training sessions during the first 36 weeks than in the last 10 weeks of the season $(3.8 v 3.3$, $\mathrm{p}<0.01$ ).

The World Cup players had an even distribution of total exposure (matches + training sessions) throughout the season, whereas the non-World Cup players had a greater total exposure during the first 36 weeks than during the last 10 weeks ( $5.6 v 4.9$ hours per week, $\mathrm{p}<0.001)$.

Table 5 shows the injury incidences during the last 10 weeks of the season compared with the first 36 weeks of the season. The injury risk at the end of the season was the same as for the rest of the season for all the players.

\section{Analysis of the 65 World Cup players}

During the last 10 weeks of the season (1 March to 15 May), the 65 players who later participated in the World Cup played a mean of 11 (4) matches (range 1-19). Nineteen (29\%) of the players incurred injuries during the World Cup, and 21 $(32 \%)$ underperformed.

The players who underperformed at the World Cup had played a mean of 12.5 matches during the 10 weeks before the event, whereas those who performed above expectation had only played nine matches $(\mathrm{p}<0.05)$ (fig 1 ).

Thirty eight of the 65 players $(61 \%)$ had played more than one match a week during the last 10 weeks of the season, and $61 \%(23 / 38)$ of these players either incurred an injury or underperformed during the World Cup (eight players from the Italian World Cup team, seven from France, four from Spain, and four from England).

\section{DISCUSSION}

This study shows that there are considerable differences in the number of matches played per season in the various leagues throughout Europe. Spanish and English teams play more league matches than Italian and French teams. The Spanish and English teams had 65-76 matches during the season, compared with less than 50 matches for the French teams.

There is a real risk of playing too many matches. In addition to the normal league matches, successful teams often take part in national and international cups, and the players also play for their country. There is reason to believe that too many matches can lead to lack of motivation and mental burn out - that is, players are no longer able to gear themselves up for matches and training sessions. ${ }^{19}$ Concentration deteriorates which can affect coordination, leading to underperformance and greater risk of injury. ${ }^{20}$ It may be that the major stress factor is not the 90 minutes of the match itself, but the mental preparation for matches, travel, and possible adaptation to changes in time and climate. ${ }^{13}$

Even though some clubs had 60-76 A team matches during a season, the number of matches played by individual players was much lower, a mean of 36 matches per player per season. So even though there are large differences in the number of matches played each season by clubs in different countries, there is very little difference between the countries with respect to the number of matches played by individual players.

Table 3 Injury incidence during the 2001-2002 season (July 2001 to May 2002)

\begin{tabular}{|c|c|c|c|c|c|}
\hline & \multicolumn{2}{|c|}{ World Cup players ( $n=65$ ) } & \multicolumn{2}{|c|}{ Non-World Cup players $(n=201)$} & \multirow[b]{2}{*}{ p Value } \\
\hline & Mean (SD) & $95 \% \mathrm{Cl}$ & Mean (SD) & $95 \% \mathrm{Cl}$ & \\
\hline Total & $7.9(4.1)$ & 7.7 to 11.6 & 9.5 (3.7) & 9.5 to 12.8 & NS \\
\hline Training & $3.2(2.2)$ & (2.3 to 4.5 & $5.5(2.1)$ & 5.6 to 8.5 & $<0.01$ \\
\hline Matches & $26.7(15)$ & 26.7 to 46.8 & $30.3(13.9)$ & 26.5 to 38.2 & NS \\
\hline
\end{tabular}


Table 4 Number of training sessions and matches per week, and exposure to football per week during the last 10 weeks of the 2001-2002 season (March 2002 to 15 May 2002) compared with the first 36 weeks of the season (July 2001 to 28 February 2002)

\begin{tabular}{|c|c|c|c|c|c|}
\hline & \multicolumn{2}{|c|}{ Last 10 weeks } & \multicolumn{2}{|c|}{ First 36 weeks } & \multirow[b]{2}{*}{ p Value } \\
\hline & Mean (SD) & $95 \% \mathrm{Cl}$ & Mean (SD) & $95 \% \mathrm{Cl}$ & \\
\hline \multicolumn{6}{|c|}{ Training sessions/week (No/player) } \\
\hline World Cup players & $4.0(0.8)$ & 3.8 to 4.2 & $3.9(0.8)$ & 3.7 to 4.1 & NS \\
\hline Non-World Cup players & $3.3(1.8)$ & 3.1 to 3.6 & $3.8(1.3)$ & 3.7 to 4.0 & $<0.001$ \\
\hline \multicolumn{6}{|l|}{ Matches/week (No/player) } \\
\hline World Cup players & $1.12(0.41)$ & 1.01 to 1.22 & $0.97(0.28)$ & 0.90 to 1.04 & $<0.01$ \\
\hline Non-World Cup players & $0.66(0.50)$ & 0.59 to 0.73 & $0.72(0.34)$ & 0.67 to 0.77 & $<0.05$ \\
\hline \multicolumn{6}{|c|}{$\begin{array}{l}\text { Exposure for training/week (hours/ } \\
\text { player) }\end{array}$} \\
\hline World Cup players & $5.1(1.2)$ & 4.8 to 5.4 & $5.1(1.0)$ & 4.8 to 5.3 & NS \\
\hline Non-World Cup players & $4.1(2.3)$ & 3.8 to 4.4 & $4.8(1.5)$ & 4.6 to 5.0 & $<0.001$ \\
\hline \multicolumn{6}{|c|}{$\begin{array}{l}\text { Exposure for matches/week (hours/ } \\
\text { player) }\end{array}$} \\
\hline World Cup players & $1.43(0.65)$ & 1.27 to 1.60 & $1.24(0.42)$ & 1.14 to 1.34 & $<0.01$ \\
\hline Non-World Cup players & $0.79(0.64)$ & 0.70 to 0.87 & $0.85(0.45)$ & 0.79 to 0.91 & $<0.05$ \\
\hline \multicolumn{6}{|c|}{ Total exposure/week (hours/player) } \\
\hline World Cup players & $6.5(1.4)$ & 6.2 to 6.9 & $6.3(1.2)$ & 6.0 to 6.6 & NS \\
\hline Non-World Cup players & $4.9(2.8)$ & 4.5 to 5.3 & $5.6(1.8)$ & 5.4 to 5.9 & $<0.001$ \\
\hline
\end{tabular}

Many big clubs have large squads of skilful players, allowing rotation to avoid overplay. In some cases rotation is unintentional, caused by injury or underperformance. The performance of such clubs is not necessarily affected if a large number of players are injured because they have many players to choose from. However, during the final period of the season, the situation may be different. At the end of the league season and in the final matches of the Champions League and UEFA Cup, teams naturally want to perform their best. They may therefore play all their star players even if there is a risk of mental exhaustion and injury.

The 65 players in our study group who participated in the World Cup played significantly more matches during the season than those who did not. The World Cup players also played significantly more matches a week during the last 10 weeks of the season than during the first 36 weeks of the season. However, the injury risk was no higher for the World Cup players than the non-World Cup players. In fact, the World Cup players had a lower injury risk at training than the non-World Cup players. Even though the World Cup players played more matches during the last 10 weeks than during the rest of the season, the risk of injury did not increase during this period of intense match play.

Table 5 Injury incidence (number of injuries/1000 hours of exposure) during the last 10 weeks of the 20012002 season (March 2002 to 15 May 2002) compared with the first 36 weeks of the season (July 2001 to 28 February 2002)

\begin{tabular}{llll}
\hline & Last 10 weeks & First 36 weeks & p Value \\
\hline Total & & & \\
All players & $10.0(5.7)$ & $9.2(2.9)$ & NS \\
$\quad$ World Cup players & $10.0(7.8)$ & $7.4(3.8)$ & NS \\
$\quad$ Non-World Cup players & $9.7(5.8)$ & $9.5(3.5)$ & NS \\
Training & $4.6(3.3)$ & $5.2(2.0)$ & NS \\
All players & $4.1(4.2)$ & $2.9(2.1)$ & NS \\
World Cup players & $4.9(3.2)$ & $5.7(2.2)$ & NS \\
$\quad$ Non-World Cup players & & & \\
Matches & $34.5(17.9)$ & $28.9(11.7)$ & NS \\
All players & $31.1(21.0)$ & $25.8(15.9)$ & NS \\
World Cup players & $34.8(24.7)$ & $29.3(14.6)$ & NS \\
Non-World Cup players & 3.9 & \\
\hline Values are mean (SD). & & & \\
\hline
\end{tabular}

This suggests that, under normal circumstances, players are able to cope with such an intensive programme because they know that a period of rest follows. However, every four years this rest period is replaced by another intense match series - the World Cup. In 2002, the first World Cup matches were played only two weeks after the Champions League final, which may explain why a number of European players underperformed in Korea/Japan. We found that 29\% of the players from our study incurred injuries during the World Cup and that $32 \%$ performed below their normal standard.

The players who underperformed in the World Cup had played an average of 12 matches during the last 10 weeks of the season compared with nine matches for the players who performed better than expected. Furthermore, we found that almost two thirds of players who had played more than one match a week during the last 10 weeks of the season either incurred injuries or underperformed in the World Cup.

Our finding that the risk of injury was not increased during the last 10 weeks of the season despite the increase in the number of matches played indicates that a top player can cope with a congested match calendar for a short period. The finding that many of the players either underperformed or incurred injury during the World Cup two to six weeks after the end of the league season indicates that the congested match calendar at the end of the season may leave the players fatigued, increasing the risk of injury and underperformance during the following period. As a result of the 2002 World

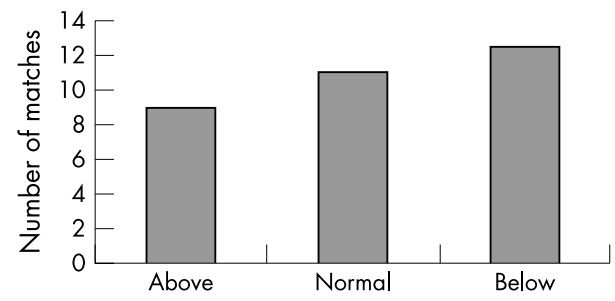

Figure 1 Number of matches played during the last 10 weeks of the ordinary season (1 March to 15 May 2002) for the 65 players who participated in the World Cup in Korea/Japan (31 May to 30 June 2002). The players are divided into three groups according to their performance during the World Cup: those who performed above or below their normal standard and those who performed as expected (normal). 


\section{Information box}

- There is a considerable variation in the number of matches played per season in European professional football leagues. Top level players are obliged to play many matches especially during the final period of the season.

- A period of match congestion can lead to player fatigue, which may result in injury and/or underperformance during the following period.

Cup experience, UEFA decided to reduce the format of the Champions League from the 2003/04 season onwards.

\section{Limitations of the study}

One weakness of the study is the limited number of teams and players included. The study involved a total of 11 teams from five nations, and 65 players who participated in the World Cup 2002. To obtain a clearer picture of the overall situation in Europe, an expanded study including more countries and teams is suggested.

Another potential weakness is the subjective evaluation of player performance during the World Cup. The three international experts were all former coaches for European national teams with great experience in evaluating the performance of players. The independent evaluations were subjective but the assessments turned out to be almost identical for most players. However, a few players had limited match exposure during the World Cup, making performance classification difficult in some cases.

Finally, the lack of exposure data for training sessions during the World Cup preparation and tournament means that no conclusions can be drawn about the risk of injury during the World Cup.

\section{CONCLUSIONS}

There is a considerable variation in the number of matches played per season in European professional leagues. Top level players are obliged to play many matches, especially during the final period of the season. This study indicates that a period with a congested match calendar can lead to fatigue, increasing the risk of injury and poor performance during the following period.

\section{ACKNOWLEDGEMENTS}

We gratefully acknowledge UEFA, the Swedish Football Association, and the Swedish Sports Confederation (Sports Research Council) for financial support. We appreciate the support and cooperation from UEFA especially from the President Lennart Johansson, the Technical Director Andy Roxburgh, the Chairman of the Medical Committee Dr
Urs Vogel, the Chairman of the Medical Committee of FIFA Dr Michel D'Hooghe, the Manager of Medical Matters of UEFA Andrea Freely, the member of the UEFA Medical Committee Dr Mogens Kreutzfeldt, the member of the Technical Instructors Panel Lars Arnesson and the former Manager of the Swedish National Team Tommy Svensson. We would also like to express our gratitude to all the team doctors and contact persons, for collection of exposure and injury data, as well as all the players and coaches for their participation in the study.

\section{Authors' affiliations}

J Ekstrand, M Waldén, M Hägglund, Department of Social Medicine and Public Health Science, Linköping University, Sweden

\section{REFERENCES}

1 Drawer S, Fuller CW. Evaluating the level of injury in English professional football using a risk based assessment process. Br J Sports Med 2002;36:446-51.

2 Hawkins RD, Fuller CW. An examination of the frequency and severity of injuries and incidents at three levels of professional football. BrJ Sports Med 1998;32:326-32.

3 Hawkins RD, Fuller CW. A prospective epidemiological study of injuries in four English professional football clubs. Br J Sports Med 1999;33:196-203.

4 Hawkins RD, Hulse MA, Wilkinson C, et al. The association football medical research programme: an audit of injuries in professional football. $\mathrm{Br} J$ Sports Med 2001;35:43-7.

5 Woods C, Hawkins R, Hulse M, et al. The Football Association Medical Research Programme: an audit of injuries in professional football-analysis of preseason injuries. Br J Sports Med 2002;36:436-41.

6 Woods C, Hawkins R, Hulse M, et al. The Football Association Medical Research Programme: an audit of injuries in professional football: an analysis of ankle sprains. Br J Sports Med 2003;37:233-8.

7 Ekstrand J. Soccer injuries and their prevention. Linköping, Sweden: Linköping University, 1982.

8 Ekstrand J. Injury prevention. In: Ekblom B, ed. Football (soccer). Oxford: Blackwell Scientific Publications, 1994:209-15

9 Ekstrand J, Gillquist J, Liljedahl SO. Prevention of soccer injuries. Supervision by doctor and physiotherapist. Am J Sports Med 1983;11:116-20.

10 Junge $\mathbf{A}$, Rosch D, Peterson L, et al. Prevention of soccer injuries: a prospective intervention study in youth amateur players. Am J Sports Med 2002;30:652-9.

11 Ekstrand J, Gillquist J. The avoidability of soccer injuries. Int J Sports Med 1983:4:124-8.

12 Ekstrand J, Gillquist J. Soccer injuries and their mechanisms: a prospective study. Med Sci Sports Exerc 1983;15:267-70.

13 Ekstrand J, Karlsson J, Hodson A. Football medicine. London: Martin Dunitz (Taylor \& Francis Group), 2003:562.

14 Hawkins RD, Fuller CW. Risk assessment in professional football: an examination of accidents and incidents in the 1994 World Cup finals. Br J Sports Med 1996;30:165-70.

15 Inklaar H. Soccer injuries. II. Aetiology and prevention. Sports Med 1994;18:81-93.

16 Mechelen Wv, Hlobil H, Kemper H. Incidence, severity, aetiology and prevention of sports injuries. Sports Med 1992;14:82-99.

17 Ekstrand J, Gillquist J, Moller M, et al. Incidence of soccer injuries and their relation to training and team success. Am J Sports Med 1983;11:63-7.

18 Ekstrand J, Karlsson J. The risk for injury in football. There is a need for a consensus about definition of injury and the design of studies. Scand J Med Sci Sports 2003;13:147-9.

19 Kentta G, Hassmen P, Raglin JS. Training practices and overtraining syndrome in Swedish age-group athletes. Int J Sports Med 2001;22:460-5.

20 Kibler WB, Chandler TJ, Stracener ES. Musculoskeletal adaptations and injuries due to overtraining. Exerc Sport Sci Rev 1992;20:99-126. 